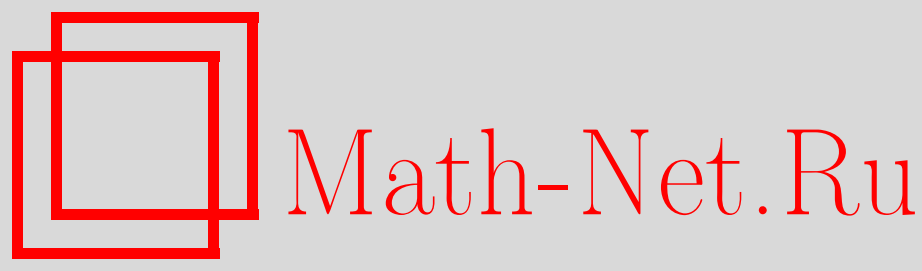

М. Данчо, М. Гнатич, Т. Лучивянски, Л. Мижишин, Критическое поведение процесса перколяции под влиянием случайного поля скоростей: однопетлевое приближение, ТМФ, 2013, том 176, номер 1, 79-88

DOI: https://doi.org/10.4213/tmf8492

Использование Общероссийского математического портала Math-Net.Ru подразумевает, что вы прочитали и согласны с пользовательским соглашением http://www.mathnet.ru/rus/agreement

Параметры загрузки:

IP : 3.82 .47 .9

26 апреля 2023 г., 06:14:03

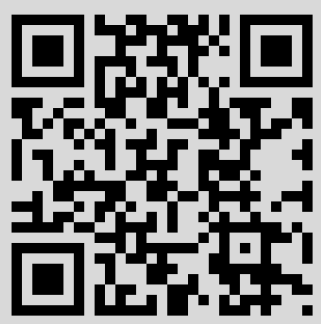




\title{
ФИЗИКА
}

Том 176, № 1

июль, 2013

(C) 2013 г.

М. Данчо*, М. Гнатич*†, Т. Лучивянски*†, Л. Мижишин ${ }^{\dagger}$

\section{КРИТИЧЕСКОЕ ПОВЕДЕНИЕ ПРОЦЕССА ПЕРКОЛЯЦИИ ПОД ВЛИЯНИЕМ СЛУЧАЙНОГО ПОЛЯ СКОРОСТЕЙ: ОДНОПЕТЛЕВОЕ ПРИБЛИЖЕНИЕ}

\begin{abstract}
С помощью пертурбативной ренормгруппы исследуется влияние случайного поля скоростей на критическое поведение направленного процесса перколяции вблизи фазового перехода второго порядка между абсорбирующей и активной фазами. Для описания поля скоростей переноса используется модель Антонова-Крейчнана с конечным временем корреляции. С целью получения информации об асимптотическом поведении рассматриваемой модели на больших масштабах применен подход теоретико-полевой ренормгруппы. Модель анализируется вблизи критической размерности с помощью трехпараметрического разложения по $\epsilon, \delta, \eta$, где $\epsilon$ - отконение от колмогоровского масштаба, $\delta$ отклонение от критической размерности пространства, а $\eta$ - отклонение от параболического закона дисперсии для коррелятора скоростей. Фиксированные точки с соответствующими областями устойчивости определяются в ведущем порядке теории возмущений.
\end{abstract}

Ключевые слова: пертурбативная ренормгруппа, направленная перколяция, турбулентная диффузия.

DOI: $10.4213 / \operatorname{tmf} 8492$

\section{1. ВВЕДЕНИЕ}

Процессы перколяции представляют собой известные модели описания случайных структур [1], [2]. Некоторые из этих процессов известны как направленная перколяция со связями (НПС). Общим свойством НПС оказывается то, что агент (частица) может распространяться от одного положения к другому по разрешенному направлению прохода. Направление распространения агентов определяет выделенное направление в пространстве. НПС может также служить для объясне-

*Institute of Experimental Physics of the Slovak Academy of Sciences, Košice, Slovakia. E-mail: danco@saske.sk

${ }^{\dagger}$ Faculty of Science, P. J. Šafárik University, Košice, Slovakia 
ния адронного взаимодействия при очень высоких энергиях (теория поля реджеона) [3], различных моделей распространения болезней [1], [2], стохастических процессов реакции-диффузии на решетке [4] или, как в первоначальной формулировке [5], смачивания пористого материала или поиска пути в лабиринте. Верхняя критическая размерность в этой задаче оценивается как $d=4$ в отличие от значения $d=6$ для случая изотропной динамики [1], [6], [7].

Одно из наиболее важных свойств НПС - появление неравновесного фазового перехода второго порядка между абсорбирующей и активной фазами. Абсорбирующая (неактивная) фаза соответствует случаю, когда в среде не содержится агентов (зараженных индивидумов), а активная фаза представляет поведение системы, когда число агентов флуктуирует вокруг постоянного значения.

Большие усилия прилагались к исследованию различных эффектов. Например, дальнодействующие взаимодействия посредством полетов Леви изучались и в пространственных, и во временны́х переменных в работах [8], введение иммунизации рассматривалось в работах [9], поверхностный эффект исследовался в работе [10] и т. д. Легко представить, что распространение болезни может ускориться вследствие наличия некоторого внешнего атмосферного течения или из-за летающих насекомых. В обоих случаях добавочный дрейф можно моделировать с помощью случайного поля скоростей [11] с заданными статистическим свойствами. В статье [11] изучается влияние адвективного поля, которое описывается моделью Крейчнана с коррелятором поля скоростей в форме белого шума. Обобщая данный подход, можно изучать, например, эффект сжимаемости [12] или, используя стохастические уравнения Навье-Стокса, эффекты "реального" турбулентного поля [13]. В настоящей работе мы исследуем влияние поля скоростей с конечной корреляцией (вводимые понятия см. в работах [14]) и определяем, каким образом оно может изменить критическое поведение процесса перколяции. Используя ренормгрупповой подход, мы определяем возможные фиксированные точки и соответствующие области устойчивости. Мы показываем, что модель имеет двенадцать возможных крупномасштабных режимов, а воздействие флуктуаций скоростей иллюстрируется на таких величинах, как число частиц, вероятность выживания активного кластера и радиус гирации активных частиц.

Статья построена следующим образом. В разделе 2 дано краткое описание модели. В разделе 3 доказана мультипликативная ренормируемость модели и в однопетлевом приближении вычислены ренормгрупповые функции (бета-функции и аномальные размерности), в разделе 4 перечислены возможные крупномасштабные режимы, а их физический смысл обсуждается в разделе 5.

\section{2. ТЕОРЕТИКО-ПОЛЕВАЯ ФОРМУЛИРОВКА МОДЕЛИ}

Существуют два подхода к теоретико-полевой формулировке НПС. Первый подход является более строгим и основывается на использовании управляющего уравнения, которое можно переписать, задействовав формализм Доя [15], в виде зависящего от времени уравнения Шредингера с неэрмитовым гамильтонианом. При переходе к непрерывному пределу получается эффективное действие, которое можно анализировать обычными теоретико-полевыми методами. Действие для чистой задачи НПС [1] можно записать в следующем виде:

$$
S_{1}=\psi^{\dagger}\left(-\partial_{t}+D_{0} \nabla^{2}-D_{0} \tau_{0}\right) \psi+\frac{D_{0} \lambda_{0}}{2}\left[\left(\psi^{\dagger}\right)^{2} \psi-\psi^{\dagger} \psi^{2}\right]
$$


где подразумеваются все требуемые интегрирования по пространственно-временным переменным. Здесь $\psi \equiv \psi(t, \mathbf{x})$ - крупнозернистая плотность зараженных индивидуумов (агентов), $\psi^{\dagger}-$ функция отклика, $D_{0}-$ константа диффузии, $\lambda_{0}-$ положительная константа связи, а $\tau_{0}$ - отклонение от порогового значения введенной вероятности. Можно предположить, что $\tau_{0} \sim p_{\mathrm{c}}-p$, где $p_{\mathrm{c}}-$ критическая вероятность перколяции (аналогично отклонению от критической температуры для равновесных моделей).

Легко показать [1], что второй подход, основанный на использовании уравнения Ланжевена, после подходящего масштабирования приводит к такому же крупнозернистому функционалу действия (1). Оба этих подхода дают одинаковые предсказания универсальных величин.

Агенты могут рассматриваться как пассивная скалярная величина [14], которую переносит поле скоростей без обратного воздействия на само поле, с нетривиальными взаимодействиями, заданными кубическими членами в (1). Включение поля скоростей $\mathbf{v}(t, \mathbf{x})$ соответствует замене в соотношении (1) частной производной по времени конвективной производной $\nabla_{t}=\partial_{t}+(\mathbf{v} \cdot \nabla)$. В отличие от работы [11] мы предполагаем, что поле скоростей - случайная гауссова величина с нулевым средним и следующим коррелятором [14]:

$$
\left\langle v_{i}(t, \mathbf{x}) v_{j}\left(t^{\prime}, \mathbf{x}^{\prime}\right)\right\rangle=\int \frac{d \omega}{2 \pi} \int \frac{d \mathbf{k}}{(2 \pi)^{d}} P_{i j}(k) D_{v}(\omega, k) e^{-i \omega\left(t-t^{\prime}\right)+i \mathbf{k}\left(\mathbf{x}-\mathbf{x}^{\prime}\right)},
$$

где $P_{i j}(k)=\delta_{i j}-k_{i} k_{j} / k^{2}$ - поперечный проектор, а $D_{v}$ имеет вид [14]

$$
D_{v}(\omega, k)=\frac{g_{10} D_{0}^{3} k^{4-d-2 \epsilon-\eta}}{\omega^{2}+u_{10}^{2} D_{0}^{2}\left(k^{2-\eta}\right)^{2}},
$$

где $g_{10}$ - константа связи, а $\epsilon, \eta, \delta$ играют роль малых параметров разложения. В настоящей статье мы понимаем $\epsilon$ как отклонение от колмогоровского скейлинга [16], а $\delta$ - как отклонение от размерности пространства четыре: $d=4-2 \delta$. Показатель $\eta$ связан с частотой $\omega \propto k^{2-\eta}$, и $\eta=4 / 3$ соответствует колмогоровской частоте. Усреднение по флуктуациям скоростей можно произвести с помощью функционала действия гауссовского типа $S_{2}=-\mathbf{v} D_{v}^{-1} \mathbf{v} / 2$.

Рассматриваемая модель (2), (3) имеет два важных предела. Первый из них известен как "модель быстрых изменений" [14], которая определяется формулами

$$
u_{10} \rightarrow \infty, \quad g_{10}^{\prime} \equiv \frac{g_{10}}{u_{10}^{2}}=\text { const }, \quad D_{v}(\omega, \mathbf{k}) \rightarrow g_{10}^{\prime} D_{0} k^{-d-2 \epsilon+\eta} .
$$

Видно, что скорости некоррелированны (белый шум) по времени. Второй предел называется пределом замороженного поля скоростей [14] и получается при

$$
u_{10} \rightarrow 0, \quad g_{0}^{\prime \prime}=\frac{g_{10}}{u_{10}}=\text { const }, \quad D_{v}(\omega, \mathbf{k}) \rightarrow g_{10}^{\prime \prime} D_{0}^{2} k^{-d+2-2 \epsilon} \pi \delta(\omega) .
$$

В этом случае коррелятор скоростей не зависит от времени в $t$-представлении.

Полная модель эквивалентна сумме функционалов, соответствующих НПС и полю скоростей:

$$
S_{0}=\psi^{\dagger}\left[-\partial_{t}-(\mathbf{v} \cdot \nabla)+D_{0} \nabla^{2}-D_{0} \tau_{0}\right] \psi+\frac{D_{0} \lambda_{0}}{2}\left[\left(\psi^{\dagger}\right)^{2} \psi-\psi^{\dagger} \psi^{2}\right]-\frac{1}{2} \mathbf{v} D_{v}^{-1} \mathbf{v} .
$$


ТАБлицА 1. Канонические размерности полей и затравочных параметров действия (4).

\begin{tabular}{|c|c|c|c|c|c|c|c|c|c|c|}
\hline$F$ & $\psi$ & $\psi^{\dagger}$ & $\mathbf{v}$ & $D_{0}$ & $\tau_{0}$ & $\lambda_{0}$ & $g_{10}$ & $g_{20}$ & $u_{10}$ & $u_{20}$ \\
\hline$d_{F}^{k}$ & $d / 2$ & $d / 2$ & -1 & -2 & 2 & $\delta$ & $2 \epsilon+\eta$ & $2 \delta$ & $\eta$ & 0 \\
\hline$d_{F}^{\omega}$ & 0 & 0 & 1 & 1 & 0 & 0 & 0 & 0 & 0 & 0 \\
\hline$d_{F}$ & $d / 2$ & $d / 2$ & 1 & 0 & 2 & $\delta$ & $2 \epsilon+\eta$ & $2 \delta$ & $\eta$ & 0 \\
\hline
\end{tabular}

Пользуясь теорией возмущений, можно легко показать [11], что параметр разложения есть $\lambda_{0}^{2}$, а не $\lambda_{0}$, что является следствием так называемой симметрии обращения быстроты (на языке теории поля реджеона)

$$
\psi(t, \mathbf{x}) \rightarrow-\psi^{\dagger}(-t, \mathbf{x}), \quad \psi^{\dagger}(t, \mathbf{x}) \rightarrow-\psi(-t, \mathbf{x})
$$

Таким образом, имеет смысл ввести новый заряд $g_{20}=\lambda_{0}^{2}$.

\section{3. КАНОНИЧЕСКИЕ РАЗМЕРНОСТИ И УЛЬТРАФИОЛЕТОВЫЕ РАСХОДИМОСТИ}

Теоретический анализ ультрафиолетовых расходимостей основан на стандартном подсчете степеней [17], [18]. В динамических моделях присутствуют два независимых масштаба: времени (частоты) и длины (импульса). Поэтому каноническая размерность любой величины $F$ определяется двумя размерностями: частоты $d_{F}^{\omega}$ и импульса $d_{F}^{k}$. Эти размерности находятся из обычных условий нормировки

$$
d_{\omega}^{\omega}=-d_{t}^{\omega}=1, \quad d_{k}^{k}=-d_{k}^{k}=1, \quad d_{k}^{\omega}=-d_{\omega}^{k}=0
$$

и требования безразмерности действия (4) (относительно размерностей импульса и частоты по отдельности). Полная каноническая размерность дается соотношением $d_{F}=d_{F}^{k}+2 d_{F}^{\omega}$ (чтобы в свободной теории получался параболический закон дисперсии $\partial_{t} \propto \nabla^{2}$ ). Она играет в теории ренормировки такую же роль, как размерность длины (импульса) в статической теории [17]. Канонические размерности модели (4) приведены в табл. 1. Модель является логарифмической при размерности пространства $d=4$ (или $\delta=0$ ) и $\epsilon=\eta=0$. Ультрафиолетовые расходимости в схеме минимальных вычитаний реализуются как полюсы по $\epsilon, \delta, \eta$ или их линейной комбинации.

Полная каноническая размерность $d_{\Gamma_{N}}$ произвольной одночастично-неприводимой функции Грина $\Gamma_{N} \equiv\langle\Phi \ldots \Phi\rangle$ задается следующим соотношением [17], [18]: $d_{\Gamma_{N}}=d+2-N_{\Phi} d_{\Phi}$, где $N_{\phi}=\left\{N_{v}, N_{\psi}, N_{\psi^{\dagger}}\right\}$ - число полей, входящих в функцию Грина $\Gamma_{N}$, и подразумевается суммирование по всем типам полей $\Phi$. В логарифмической теории поверхностные ультрафиолетовые расходимости могут затрагивать только такие функции $\Gamma_{N}$, для которых $d_{\Gamma_{N}}-$ неотрицательное целое число. Функции Грина должны содержать оба поля $\psi$ и $\psi^{\dagger}$, поскольку функции $\langle\psi \ldots \psi\rangle$ или $\left\langle\psi^{\dagger} \ldots \psi^{\dagger}\right\rangle$ включают замкнутые петли запаздывающих пропагаторов [11], [17]. Поэтому поверхностные ультрафиолетовые расходимости могут встречаться только 
в функциях

$$
\begin{array}{lrl}
\left\langle\psi^{\dagger} \psi\right\rangle & \text { с контрчленами } & \psi^{\dagger} \partial_{t} \psi, \psi^{\dagger} \partial^{2} \psi, \psi^{\dagger} \psi, \\
\left\langle\psi^{\dagger} \psi \psi\right\rangle & \text { с контрчленом } & \psi^{\dagger} \psi^{2}, \\
\left\langle\psi^{\dagger} \psi^{\dagger} \psi\right\rangle & \text { с контрчленом } & \left(\psi^{\dagger}\right)^{2} \psi, \\
\left\langle\psi^{\dagger} \psi v\right\rangle & \text { с контрчленом } & \psi^{\dagger}(v \partial) \psi, \\
\left\langle\psi^{\dagger} \psi v v\right\rangle & \text { с контрчленом } & \psi^{\dagger} \psi v v .
\end{array}
$$

Члены $\left(\psi^{\dagger}\right)^{2} \psi$ и $\psi^{\dagger} \psi^{2}$ можно ренормировать с помощью той же константы ренормировки вследствие симметрии (5), а контрчлен, порожденный функцией Грина $\left\langle\psi^{\dagger} \psi v\right\rangle$, сводится к виду $\psi^{\dagger}(v \partial) \psi=-\psi(v \partial) \psi^{\dagger}$ из-за поперечности поля скоростей. В модели (быстрых изменений) Крейчнана контрчлен $\psi^{\dagger} \psi v v$ отсутствует, и константы ренормировки для членов $\psi^{\dagger} \partial_{t} \psi$ и $\psi^{\dagger}(v \partial) \psi$ остаются такими же (конвективное дифференцирование $\nabla_{t}=\partial_{t}+(\mathbf{v} \cdot \nabla)$ сохраняет форму в процессе ренормировки) в силу галилеевой инвариантности модели. Необходимо подчеркнуть, что наша модель не является галилеево-инвариантной вследствие вида коррелятора скоростей (3), что отражает существование эффекта сноса в развитой турбулентности [17]. Как следствие константы ренормировки при членах $\psi^{\dagger} \partial_{t} \psi$ и $\psi^{\dagger}(v \partial) \psi$ в этом случае другие. Более того, чтобы гарантировать мультипликативную ренормируемость модели, приходится добавить новый член $\psi^{\dagger} \psi v v$ в действие (4) с новым независимым параметром (зарядом) $u_{2}$, который компенсирует расходимость, порожденную корреляционной функцией $\left\langle\psi^{\dagger} \psi v v\right\rangle$. Полученное ренормированное действие можно переписать в следующем виде:

$$
\begin{aligned}
S_{\mathrm{R}}= & \psi^{\dagger}\left(-Z_{1} \partial_{t}-Z_{2}(\mathbf{v} \cdot \nabla)+Z_{3} D \nabla^{2}-Z_{4} D \tau\right) \psi+ \\
& +Z_{5} \frac{D \lambda}{2}\left[\left(\psi^{+}\right)^{2} \psi-\psi^{+} \psi^{2}\right]+Z_{6} \frac{u_{2}}{2 D} \psi^{\dagger} \psi \mathbf{v}^{2}-\frac{1}{2} \mathbf{v} D_{v}^{-1} \mathbf{v} .
\end{aligned}
$$

Заметим, что из соображений размерности заряд $u_{2}$ должен фигурировать в комбинации $u_{2} / D$, иначе у него будет ненулевая размерность относительно пространственной и временной переменных. С другой стороны, ренормированное действие можно получить с помощью мультипликативной ренормировки полей $\psi \rightarrow \psi Z_{\psi}$, $\psi^{\dagger} \rightarrow \psi^{\dagger} Z_{\psi^{\dagger}}, v \rightarrow v Z_{v}$ и ренормировки параметров

$$
\begin{gathered}
D_{0}=D Z_{D}, \quad g_{10}=g_{1} \mu^{2 \epsilon+\eta} Z_{g_{1}}, \quad \tau_{0}=\tau Z_{\tau}, \quad u_{10}=u_{1} \mu^{\eta} Z_{u_{1}}, \\
\lambda_{0}=\lambda \mu^{\delta} Z_{\lambda}, \quad g_{20}=g_{2} \mu^{2 \delta} Z_{g_{2}}, \quad u_{20}=u_{2} Z_{u_{2}} .
\end{gathered}
$$

Константы ренормировки полей и параметров (7) связаны с константами ренормировки действия (6) следующими соотношениями:

$$
\begin{aligned}
& Z_{v}=Z_{1}^{-1} Z_{2}, \quad Z_{\psi}=Z_{\psi^{\dagger}}=Z_{1}^{1 / 2}, \quad Z_{\lambda}=Z_{1}^{-1 / 2} Z_{3}^{-1} Z_{5}, \\
& Z_{D}=Z_{1}^{-1} Z_{3}, \quad Z_{u_{1}}=Z_{1} Z_{3}^{-1}, \quad Z_{\tau}=Z_{3}^{-1} Z_{4} \text {, } \\
& Z_{g_{1}}=Z_{1}^{5} Z_{2}^{-2} Z_{3}^{-3}, \quad Z_{g_{2}}=Z_{1}^{-1} Z_{3}^{-2} Z_{5}^{2}, \quad Z_{u_{2}}=Z_{2}^{-2} Z_{3} Z_{6} \text {. }
\end{aligned}
$$

Стандартный пертурбативный подход ренормгруппы основан на фейнмановской диаграммной технике [17], [18]. Явные вычисления в однопетлевом приближении 
приводят к следующим результатам для констант ренормировки:

$$
\begin{array}{lll}
Z_{1}=1+\frac{g_{2}}{8 \delta}, & Z_{2}=1+\frac{g_{2}}{8 \delta}, & Z_{3}=1+\frac{g_{2}}{16 \delta}-\frac{3 g_{1}}{8 u(1+u) \epsilon} \\
Z_{4}=1+\frac{g_{2}}{4 \delta}, & Z_{5}=1+\frac{g_{2}}{2 \delta}, & Z_{6}=1+\frac{g_{2}}{16 \delta}-\frac{3 g_{1}\left(1+u_{2}\right)}{8 u_{1}\left(1+u_{1}\right) \epsilon}
\end{array}
$$

Запишем основное дифференциальное уравнение ренормгруппы для ренормированной функции Грина $G_{\mathrm{R}}$ :

$$
\left\{D_{\mathrm{RG}}+N_{\psi} \gamma_{\psi}+N_{\psi^{\dagger}} \gamma_{\psi^{\dagger}}\right\} G_{\mathrm{R}}(e, \mu, \ldots)=0
$$

где $e=\left\{D, \tau, u_{1}, u_{2}, g_{1}, g_{2}\right\}$ - полный набор ренормированных аналогов затравочных параметров, а многоточие означает другие параметры такие, как пространственные и временны́е переменные. Оператор ренормгруппы $D_{\mathrm{RG}}$ можно представить в виде

$$
D_{\mathrm{RG}}=\mu \partial_{\mu}+\beta_{u_{1}} \partial_{u_{1}}+\beta_{u_{2}} \partial_{u_{2}}+\beta_{g_{1}} \partial_{g_{1}}+\beta_{g_{2}} \partial_{g_{2}}-\gamma_{D} D_{D}-\gamma_{\tau} D_{\tau},
$$

где $D_{x}=x \partial_{x}$ для любой переменной $x, \gamma_{a}=\widetilde{D}_{\mu} \ln Z_{a}$ - аномальная размерность, a $\widetilde{D}_{\mu}$ обозначает дифференциальный оператор, взятый при фиксированных значениях затравочных параметров. В формуле (10) бета-функция определяется как $\beta_{g}=\widetilde{D}_{\mu} g$, где $g \in\left\{g_{1}, g_{2}, u_{1}, u_{2}\right\}$, и из соотношений (7) напрямую получаются следующие выражения:

$$
\begin{array}{ll}
\beta_{g_{1}}=g_{1}\left(-2 \epsilon-\eta+3 \gamma_{D}\right), & \beta_{g_{2}}=g_{2}\left(-2 \delta-\gamma_{g_{2}}\right), \\
\beta_{u_{1}}=u_{1}\left(-\eta+\gamma_{D}\right), & \beta_{u_{2}}=-u_{2} \gamma_{u_{2}} .
\end{array}
$$

Из явных формул (9) и соотношений (8) легко вычисляются нужные аномальные размерности:

$$
\gamma_{D}=\frac{3 g_{1}}{4 u_{1}\left(1+u_{1}\right)}+\frac{g_{2}}{8}, \quad \gamma_{g_{2}}=-\frac{3 g_{1}}{2 u_{1}\left(1+u_{1}\right)}-\frac{3 g_{2}}{2}, \quad \gamma_{u_{2}}=-\frac{g_{2}}{8}+\frac{3 g_{1}\left(1+u_{2}\right)}{4 u_{1}\left(1+u_{1}\right)} .
$$

\section{4. ФИКСИРОВАННЫЕ ТОЧКИ И РЕЖИМЫ СКЕЙЛИНГА}

Согласно теории ренормгруппы инфракрасное (ИК) асимптотическое поведение управляется ИК-притягивающими фиксированными точками. Фиксированные точки $g^{*}=\left\{g_{1}^{*}, g_{2}^{*}, u_{1}^{*}, u_{2}^{*}\right\}$ можно найти из требования, что в этих точках все бетафункции должны одновременно обращаться в ноль: $\beta_{g_{1}}\left(g^{*}\right)=\beta_{g_{2}}\left(g^{*}\right)=\beta_{u_{1}}\left(g^{*}\right)=$ $\beta_{u_{2}}\left(g^{*}\right)=0$. Тип фиксированной точки определяется по собственным значениям матрицы $\Omega=\left\{\Omega_{i j}=\partial \beta_{i} / \partial g_{j}\right\}$, где $\beta_{i}$ - полный набор бета-функций (11), а $g_{j}$ - полный набор зарядов $\left\{g_{1}, g_{2}, u_{1}, u_{2}\right\}$. Для ИК-притягивающих фиксированных точек собственные значения матрицы $\Omega$ - строго положительные величины. Из этого условия определяется область устойчивости для данной фиксированной точки. Хорошо известно, что в ИК-области функции Грина проявляют скейлинговое поведение. Критическая размерность $\Delta_{F}$ ИК-величины $F$ определяется следующим соотношением: $\Delta_{F}=d_{F}^{k}+\Delta_{\omega} d_{F}^{\omega}+\gamma_{F}^{*}$, где $d_{F}^{k, \omega}$ - каноническая размерность величины $F$ из табл. $1, \gamma_{F}^{*}-$ значение аномальной размерности в данной фиксированной точке, а $\Delta_{\omega}=2-\gamma_{D}^{*}$. 
ТАБлицА 2. Устойчивые фиксированные точки замороженного поля скоростей и соответствующие критические показатели.

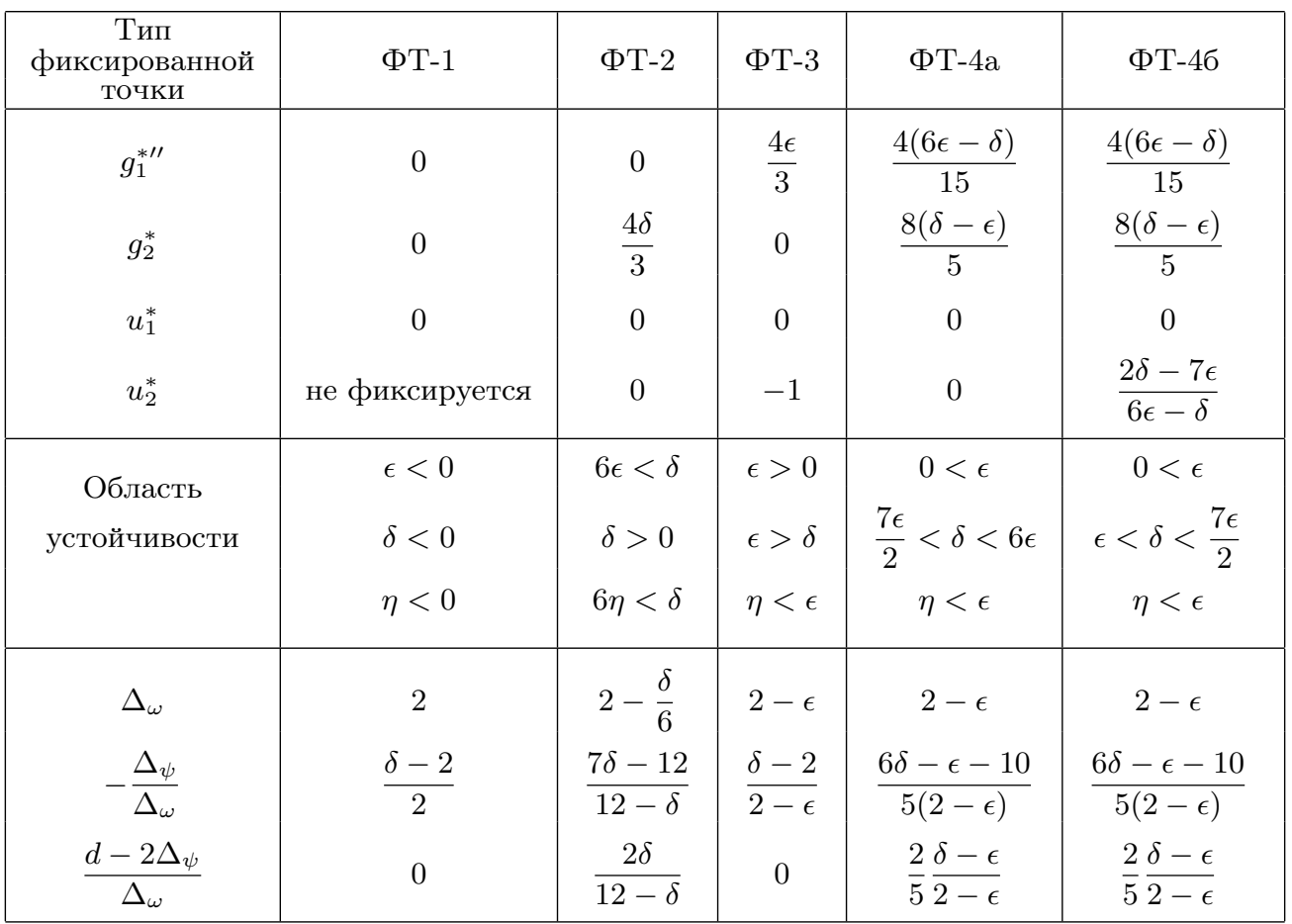

Введем теперь величины из работы [1], чтобы, возможно, прояснить структуру фиксированных точек модели. Первая величина - это число $N(t)$ активных частиц, распространяющихся из начала координат $\mathbf{x}=0$ в момент времени $t=0$, задаваемое функцией отклика:

$$
N(t)=\int d \mathbf{x}\left\langle\psi(t, \mathbf{x}) \psi^{\dagger}(0, \mathbf{0})\right\rangle \propto t^{\left(d-2 \Delta_{\psi}\right) / \Delta_{\omega}}=t^{g_{2}^{*} / 4\left(2-\gamma_{D}^{*}\right)},
$$

где $\gamma_{D}^{*}=\gamma_{D}\left(g_{1}^{*}, g_{2}^{*}, u^{*}\right)$. Вторая величина - это вероятность $P(t)$ выживания активного кластера:

$$
P(t)=-\lim _{A \rightarrow \infty}\left\langle e^{-A \psi(0, \mathbf{x})} \psi^{\dagger}(-t, \mathbf{0})\right\rangle \propto t^{-\Delta_{\psi} / \Delta_{\omega}}=t^{-\left(2-\delta-g_{2}^{*} / 8\right) /\left(2-\gamma_{D}^{*}\right)} .
$$

Радиус гирации $R(t)$ активных частиц определяется так:

$$
R^{2}(t)=\frac{\int \mathrm{d} \mathbf{x} \mathbf{x}^{2}\left\langle\psi(t, \mathbf{x}) \psi^{\dagger}(0, \mathbf{0})\right\rangle}{\int \mathrm{d} \mathbf{x}\left\langle\psi(t, \mathbf{x}) \psi^{\dagger}(0, \mathbf{0})\right\rangle} \propto t^{2 / \Delta_{\omega}}=t^{2 /\left(2-\gamma_{D}^{*}\right)}
$$

Для исследования фиксированных точек этой модели воспользуемся точным соотношением для бета-функций: $\beta_{g_{1}} / g_{1}-3 \beta_{u_{1}} / u_{1}=2(\eta-\epsilon)$. В результате получается, что бета-функции $\beta_{g_{1}}$ и $\beta_{u_{1}}$ могут одновременно обращаться в ноль при конечных значениях своих аргументов только в случае $\epsilon=\eta$, который следует изучить отдельно. С другой стороны, при $\epsilon \neq \eta$ необходимо рассматривать предельное поведение, 


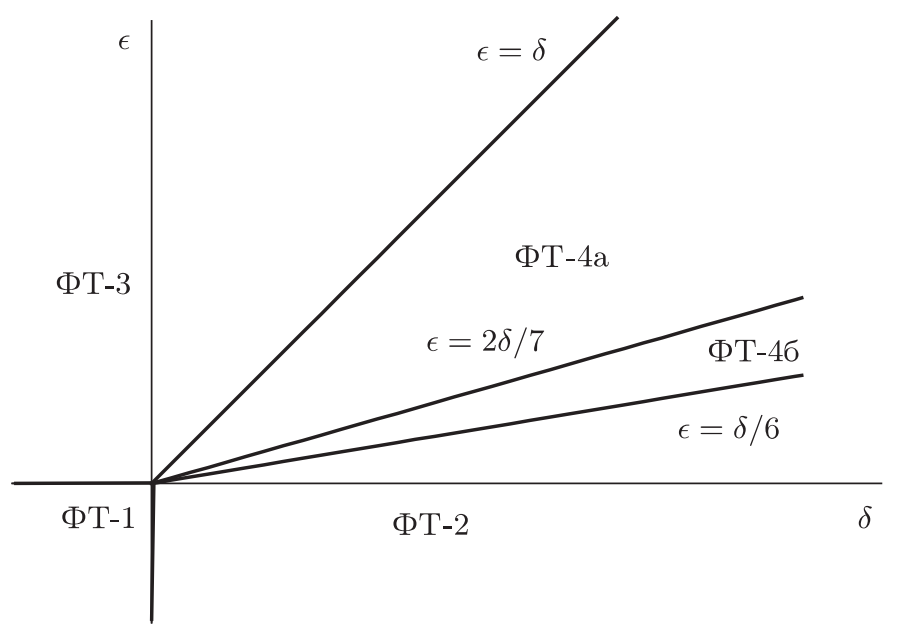

Рис. 1. Области устойчивости для фиксированных точек замороженного поля скоростей.

т. е. либо $u_{1}=0$, либо $u_{1}=\infty$, и перемасштабировать $g_{1}$ так, чтобы в бета-функциях $\beta_{g_{1}}, \beta_{u_{1}}$ аномальная размерность $\gamma_{D}$ имела конечное значение.

Рассмотрим случай, когда предел коррелятора поля скоростей отвечает известному замороженному полю скоростей и имеет четыре фиксированные точки (см. табл. 2). Для этого режима мы ввели новую переменную $g_{1}^{\prime \prime} \equiv g_{1} / u_{1}$, ее бетафункция имеет вид $\beta_{g_{1}^{\prime \prime}}=g_{1}^{\prime \prime}\left(-2 \epsilon+2 \gamma_{D}\right)$. Фиксированная точка $\Phi$ Т-1 соответствует гауссовой (свободной) фиксированной точке. В этом режиме радиус гирации $R(t)$ имитирует обычное случайное блуждание $\left(R(t) \propto t^{1 / 2}\right)$. Для фиксированной точки ФТ-2 коррелятор поля скоростей является несущественным, и модель ведет себя как "чистый" класс направленных перколяций с соответствующими критическими показателями [1]. Для фиксированной точки ФТ-3 нелинейность действия (1) процесса направленной перколяции несущественна. Наконец, фиксированные точки ФТ-4а и ФТ-4б соответствуют нетривиальному режиму ИК-скейлинга, в котором важны и нелинейность модели (1) направленной перколяции, и поле скоростей. Кроме того, для фиксированной точки ФТ-4а несущественно взаимодействие $\psi^{\dagger} \psi \mathbf{v}^{2}$, в отличие от случая фиксированной точки ФТ-4б. В интервале $\epsilon \in(0,2)$ и при $\delta=$ const показатель вероятности выживания $P(t)$ меньше -1 для фиксированной точки ФТ-3 и больше -1 для фиксированной точки $\Phi$ Т-4. Следовательно, в режиме фиксированной точки ФТ-4 вероятность выживания убывает медленнее, чем для фиксированной точки ФТ-3. В режиме фиксированной точки ФТ-3 флуктуации скорости приводят к убыванию активного кластера, а в режиме фиксированной точки ФТ-4 - к противоположному эффекту.

На рис. 1 изображены области устойчивости для фиксированных точек в плоскости $(\epsilon, \delta)$ при $\eta \leqslant 0$. Границы областей показаны жирными линиями.

Второй предельный случай характеризуется полем скоростей типа белого шума. Этот режим называется "моделью быстрых изменений" [14] и имеет четыре фиксированные точки. В этом случае полезно ввести новые переменные $g_{1}^{\prime} \equiv g_{1} / u_{1}^{2}$ и $w=1 / u_{1}$, для которых соответствующие бета-функции имеют следующий вид: 
ТАБлицА 3. Фиксированные точки в нетривиальном случае.

\begin{tabular}{|c|c|c|c|}
\hline $\begin{array}{c}\text { Фиксированной } \\
\text { точки }\end{array}$ & ФТ-5 & ФТ-6а & ФТ-6б \\
\hline$\frac{g_{1}^{*}}{u_{1}^{*}\left(1+u_{1}^{*}\right)}$ & $\frac{4 \epsilon}{3}$ & $\frac{4(6 \epsilon-\delta)}{15}$ & $\frac{4(6 \epsilon-\delta)}{15}$ \\
$g_{2}^{*}$ & 0 & $\frac{8(\delta-\epsilon)}{5}$ & $\frac{8(\delta-\epsilon)}{5}$ \\
$u_{2}^{*}$ & -1 & 0 & $\frac{2 \delta-7 \epsilon}{6 \epsilon-\delta}$ \\
\hline Область & $\epsilon>0$ & $\epsilon>0$ & $\epsilon>0$ \\
устойчивости & $\eta=\epsilon>\delta$ & $\frac{\eta}{2}=\frac{2 \delta}{7}$ & $2-\epsilon$ \\
\hline$\Delta_{\omega}$ & $2-\epsilon$ & $\frac{6 \delta-\epsilon-10}{5(2-\epsilon)}$ & $\frac{6 \delta-\epsilon-10}{5(2-\epsilon)}$ \\
$-\frac{\Delta_{\psi}}{\Delta_{\omega}}$ & $\frac{\delta-2}{2-\epsilon}$ & $\frac{2}{5} \frac{\delta-\epsilon}{2-\epsilon}$ & $\frac{2 \delta}{2-\epsilon}$ \\
\hline$\frac{d-2 \Delta_{\psi}}{\Delta_{\omega}}$ & 0 & & \\
\hline
\end{tabular}

$\beta_{g_{1}^{\prime}}=g_{1}^{\prime}\left(\eta-2 \epsilon+\gamma_{D}\right)$ и $\beta_{w}=w\left(\eta-\gamma_{D}\right)$. Фиксированные точки и их области устойчивости можно найти в работе [11].

Третий, наиболее нетривиальный, случай отличается от предыдущих: бета-функции $\beta_{g_{1}}$ и $\beta_{u_{1}}$ пропорциональны между собой, так что фиксированные точки вырождаются. Однако фиксированные точки можно разделить на две группы в соответствии со значением $g_{2}$ (см. табл. 3). Для фиксированной точки ФТ-5 нелинейность действия (1) направленной перколяции несущественна. Для фиксированных точек ФТ-6а и ФТ-6б соответствующая взаимодействию часть действия (1) и поле коррелятора скоростей сосуществуют. В интервале $\epsilon \in(0,2)$ показатели величин $N(t), P(t)$ и $R(t)$ ведут себя так же, как фиксированные точки ФТ-3, ФТ-4а и ФТ-4б соответственно.

\section{5. ЗАКЛЮЧЕНИЕ}

Мы исследовали влияние поля скоростей с конечным радиусом корреляции по времени (модель Антонова-Крейчнана) на развитие процесса направленной перколяции со связями. Построив теоретико-полевую модель, мы вычислили константы ренормировки тройного разложения по $\epsilon, 2 \delta=4-d$ и $\eta$ в ведущем порядке и нашли фиксированные точки, их области устойчивости и число активных частиц, вероятность выживания и радиус гирации.

Благодарности. Работа поддержана Ministry of Education, Science, Research and Sport of the Slovak Republic (грант VEGA 1/0222/13), Centre of Excellency for Nanofluid, Institute of Experimental Physics, Slovak Academy of Sciences. Lukáš Mižišin благодарит за поддержку VVGS грант UPJS. Эта статья создавалась в рамках программы "Cooperative phenomena and phase transition in nanosystems with perspective 
utilization in nano- and biotechnology" (проекты № 26220120033, 26110230061). Средства для исследования и программы развития предоставил European Regional Development Fund.

\section{Список литературы}

[1] H.-K. Janssen, U. C. Täuber, Ann. Phys., 315:1 (2004), 147-192, arXiv: cond-mat/0409670.

[2] U. C. Täuber, Adv. Solid State Phys., 43 (2003), 659-575.

[3] J. L. Cardy, R. L. Sugar, J. Phys. A, 13:12 (1980), L423-L427.

[4] H. Hinrichsen, Adv. Phys., 49:7 (2000), 815-958, arXiv: cond-mat/0001070.

[5] S. R. Broadbent, I. M. Hamersley, Proc. Cambr. Phil. Soc., 53:3 (1957), 629-641.

[6] H. K. Janssen, Z. Phys. B, 58:4 (1985), 311-317.

[7] E. Frey, U. C. Täuber, F. Schwabl, Phys. Rev. E, 49:6 (1994), 5058-5072.

[8] H.-K. Janssen, K. Oerding, F. van Wijland, H. J. Hilhorst, Eur. Phys. J. B, 7:1 (1999), 137-145; H. Hinrichsen, J. Stat. Mech., 07 (2007), P07006, 35 pp., arXiv: cond-mat/0702169; H.-K. Janssen, O. Stenull, Phys. Rev. E, 78:6 (2008), 061117, 12 pp., arXiv: 0809.2344).

[9] J. L. Cardy, P. Grassberger, J. Phys. A, 18:6 (1985), L267-L271; J. L. Cardy, J. Phys. A, 16:18 (1983), L709-L712; F. Linder, J. Tran-Gia, S. R. Dahmen, H. Hinrichsen, J. Phys. A, 41:18 (2008), 185005, 11 pp.

[10] H.-K. Janssen, B. Schaub, B. Schittmann, Z. Phys. B, 72:1 (1988), 111-114.

[11] N. V. Antonov, V. I. Iglovikov, A. S. Kapustin, J. Phys. A, 42:13 (2008), 135001, 19 pp., arXiv: 0808.0076.

[12] N. V. Antonov, A. S. Kapustin, J. Phys. A, 43:40 (2010), 405001, 22 pp.

[13] Н. В. Антонов, А. С. Капустин, А. В. Малышев, ТМФ, 169:1 (2011), 124-136.

[14] N. V. Antonov, Phys. Rev. E, 60:6 (1999), 6691-6707; Physica D, 144:3-4 (2000), 370-386.

[15] M. Doi, J. Phys. A, 9:9 (1976), 1456-1477; 1479-1495.

[16] У. Фриш, Турбулентность. Наследие А.Н. Колмогорова, ФАЗИС, М., 1998.

[17] А.Н. Васильев, Квантовополевая ренормгруппа в теории критического поведения и стохастической динамике, Изд-во ПИЯФ, СПб., 1998.

[18] J. Zinn-Justin, Quantum Field Theory and Critical Phenomena, International Series of Monographs on Physics, 77, Oxford Univ. Press, Oxford, 1989. 